\title{
IBAS, the INTEGRAL Burst Alert System
}

\author{
A status report \\ H. Pedersen ${ }^{5}$ \\ 1 INTEGRAL Science Data Centre, CH-1290 Versoix, Switzerland \\ 2 Istituto di Fisica Cosmica "G. Occhialini", Milano, Italy \\ 3 IFIC, Valencia, Spain \\ 4 Geneva Observatory, CH-1290 Sauverny, Switzerland \\ ${ }^{5}$ CUO, Copenhagen, Denmark
}

B. Kretschmar ${ }^{1}$, S. Mereghetti ${ }^{2}$, D. Jennings ${ }^{1}$, A. Pellizzoni ${ }^{2}$, A. Robert ${ }^{1,3}$, R. Walter ${ }^{1,4}$, T.J.-L. Courvoisier ${ }^{1,4}$, and $^{\text {. }}$

Received December 29, 1998; accepted April 21, 1999

Abstract. We report on the INTEGRAL Science Data Centre (ISDC) BURST ALERT SYSTEM, designed to automatically distribute GRB positions as measured with INTEGRAL within tens of seconds after the burst.

Key words: gamma-rays: bursts - gamma-rays: observations - instrumentation: miscellaneous

\section{Introduction}

INTEGRAL (International Gamma Ray Astrophysical Laboratory), an ESA $\gamma$-ray astronomy mission to be launched in 2001 (Winkler 1997), is expected to add significantly to the current understanding of GRBs. Its three coaligned high energy instruments (JEM-X, SPI, IBIS) use coded mask imaging technology to cover the energy range $3 \mathrm{keV}$ through $10 \mathrm{MeV}$; the optical monitor (OMC) supplements the coverage in the band of $500 \mathrm{~nm}$ to $850 \mathrm{~nm}$ (Table 1).

The good imaging capabilities of IBIS, its large field of view and high sensitivity around $100 \mathrm{keV}$, coupled with a continuous telemetry flow and a highly excentric $72 \mathrm{~h}$ orbit allow for long uninterrupted observations and permit both fast and accurate localizations of GRB events.

\section{The INTEGRAL Burst Alert System IBAS}

INTEGRAL will not have a GRB detection and triggering system on board. However, it will downlink its acquired data continuously to earth allowing for constant near-real time monitoring. At the ISDC all data will be automatically analyzed to detect any transient events. In addition, a fast analysis (Table 2) will be performed by the INTEGRAL Burst Alert System IBAS (Mereghetti et al. 1999). This "on ground" approach to detection not only allows for the application of larger computational power than available on board a spacecraft, but also permits the implementation of several detection algorithms running in parallel (Fig. 1).

Send offprint requests to: Baerbel.Kretschmar@obs.unige.ch

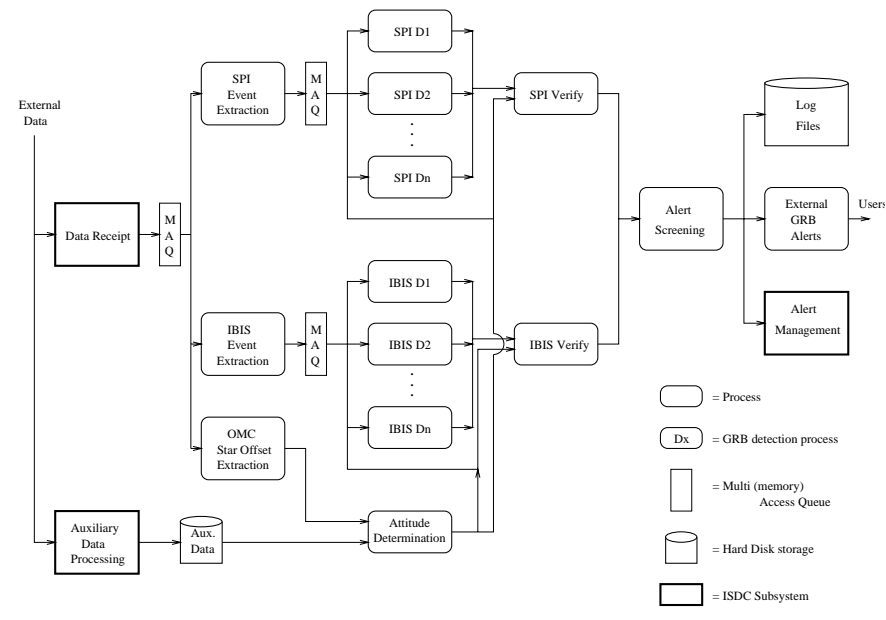

Fig. 1. The IBAS Software Architecture at ISDC

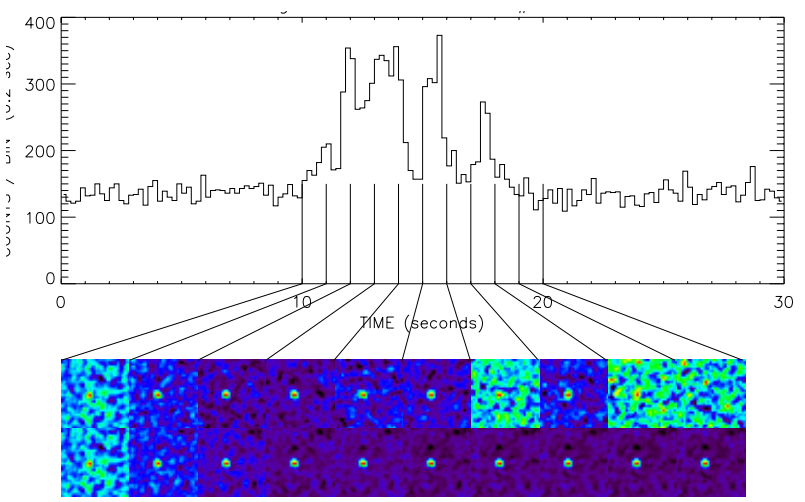

Fig. 2. Light curve and deconvolved images of a simulated GRB in the ISGRI fully coded field of view (ISGRI is a detector layer of IBIS). The upper image row displays $15-300 \mathrm{keV}$ images of $1 \mathrm{~s}$ integration each, while the images in the lower row have been obtained integrating over increasingly longer time intervals (from 1 to $10 \mathrm{~s}$ ). This shows how rapidly it is possible to obtain a good signal to noise level for a typical GRB 
Table 1. Main instrument characteristics. FOV $=$ (Partially coded) Field of View; FCFOV = Fully Coded Field of View; the angular resolution is given at FWHM; the spectral resolution is measured in $(\Delta E / E)$

\begin{tabular}{l|clllll}
\hline Instr. & Energy Range & FOV & FCFOV & Ang. Res. & Spectr. Res. & Source Loc. \\
\hline IBIS & $15 \mathrm{keV}-10 \mathrm{MeV}$ & $30^{\circ}$ & $9^{\circ}$ & $12^{\prime}$ & $<7 \%$ at $100 \mathrm{keV}$ & $\sim 1^{\prime}$ \\
SPI & $20 \mathrm{keV}-8 \mathrm{MeV}$ & $30^{\circ}$ & $16^{\circ}$ & $2^{\circ}$ & $0.2 \%$ at $1 \mathrm{MeV}$ & $<30^{\prime}$ \\
JEM-X & $3 \mathrm{keV}-35 \mathrm{keV}$ & $13.2^{\circ}$ & $4.8^{\circ}$ & $3^{\prime}$ & $<47 \%(E / 1 \mathrm{keV})^{-0.5}$ & $<30^{\prime \prime}$ \\
OMC & $500 \mathrm{~nm}-850 \mathrm{~nm}$ & $5^{\circ}$ & n.a. & $17.6^{\prime \prime}$ & & $\sim 8^{\prime \prime}$ \\
\hline
\end{tabular}

Table 2. The breakdown of the anticipated full delay with IBAS. The largest uncertainty in this chain lies in the time it will take the groundstations (GS) to transfer the spacecraft's (S/C) telemetry frames to ESA's Mission Operation Centre, and from MOC to the INTEGRAL Science Data Centre ISDC

\begin{tabular}{ll}
\hline S/C to GS to MOC to ISDC & $5<t<30 \mathrm{~s}$ (TBC) \\
Data reception and extraction & $\sim 3 \mathrm{~s}$ \\
GRB detection and triggering & $1<t<10 \mathrm{~s}$ \\
Validation and positioning & $1<t<10 \mathrm{~s}$ \\
Alert generation & $<2 \mathrm{~s}$ \\
\hline Total: & $12<t<55 \mathrm{~s}$ \\
\hline
\end{tabular}

After receiving the INTEGRAL telemetry at ISDC, the IBAS relevant data is extracted and fed into the attitude determination and into the several detection processes $D_{n}$ running in parallel. As soon as a GRB candidate event is detected, it must pass a verification process and a final screening, which is additionally in charge of spawning a more detailed offline analysis of the burst; the exact verification algorithm is still to be defined. The GRB position and trigger time then reach the alert generation process, and the information is broadcast electronically.

Ongoing IBAS simulations currently concentrate on GRB detection with IBIS, since its photon by photon mode data is expected to yield the best position accuracy. During the simulations photon arrival times are generated based on light curves actually observed by BATSE. The simulations shown in Fig. 2 e.g. reveal the GRB to be localizable less than $2 \mathrm{~s}$ after the trigger time $(S / N \sim 20)$. This has been achieved using a simplified prototype code: The countrates of the incoming events are binned, averaged in time $\left(R_{\text {bin }}, t_{\text {bin }}\right.$ and $R_{\text {avg }}, t_{\text {avg }}$ respectively) and compared, taking the deviation $\sigma$ into account. The trigger time $t_{\text {trig }}$ is recorded as soon as a threshold is passed $\left(R_{\text {bin }}>R_{\text {avg }}+n \sigma\right)$. Images are deconvolved (preburst and integrated ones over $t_{\text {image }}$ time bins) and the position is found. The simulation parameters for Fig. 2 were $n=7 ; t_{\text {bin }}=50 \mathrm{~ms} ; t_{\text {trig }}=11.7 \mathrm{~s} ; t_{\text {image }}=1 \mathrm{~s}$. It was based on BATSE trigger \# 2321 featuring a peak flux of $0.85 \mathrm{ph} /\left(\mathrm{cm}^{2} \mathrm{~s}\right)$ in the $50-300 \mathrm{keV}$ channel. A broken power law spectrum is assumed with photon index $\alpha_{1}=0.7$ and $\alpha_{2}=2.0$ and with break energy $E_{\text {break }}=100 \mathrm{keV}$.

INTEGRAL is expected to detect about 20 GRBs per year within the IBIS and SPI fields of view (Pedersen et al. 1997). Localization accuracy is a function of the event's $S / N$ ratio, the spacecraft attitude and stability, the instrument to star-tracker alignment and the instrument angular resolution. The attitude accuracy will be $\leq 30^{\prime \prime}$ during stable pointings, i.e. for most of the time (during slews it will be $\sim 10^{\prime}$ ). For a $10 \sigma$ source detected with IBIS the source location accuracy is $\sim 1^{\prime}$. The use of OMC data for improved attitude information is under study, as OMC will provide offset values $\Delta Y$ and $\Delta Z$ to the central source position with an anticipated accuracy of $<17^{\prime \prime}$.

Although SPI's localization accuracy will be significantly worse than that of IBIS, SPI data will be used to assess the validity of the event. Additionally, SPI may provide localization for those bursts at large off axis angles. For those GRBs SPI's sensitivity is better than that of IBIS due to the larger fully coded field of view.

In the relevant time frame of 2001 to 2003 INTEGRAL seems to be the satellite best suited as the Interplanetary Network's near earth node. As an optimized input to the IPN, SPI's anticoincidence shield (ACS) will take data in time bins of $50 \mathrm{~ms}$, time tagged to an accuracy of $1 \mathrm{~ms}$. Thus the data of $\sim 300(5 \sigma)$ bursts per year, located mainly perpendicular to the instruments' fields of view, can usefully contribute to the IPN (Hurley 1999; Teegarden \& Sturner 1999).

\section{Summary and conclusions}

Several detection processes running in parallel on ground will allow to trigger on GRBs of a wide range of given durations. Simulations have shown that about 20 bursts per year will be localized with arcminute accuracy only tens of seconds after their occurence. IBAS will distribute their positions in near real time via TCP/IP or e-mail to all parties interested. Additionally, INTEGRAL data of $\sim 300$ annual GRBs, via the SPI ACS, will contribute to the IPN localization.

\section{References}

Hurley K., 1999, in: Bazzano A. (ed.), Proc. 3rd INTEGRAL Workshop "The Extreme Universe", astro-ph/9812053

Mereghetti S., et al., 1999, in: Bazzano A. (ed.), Proc. 3rd INTEGRAL Workshop "The Extreme Universe"

Pedersen H., Jennings D., Mereghetti S., Teegarden B., 1997, in: Winkler C., Courvoisier T.J.-L. and Durouchoux P. (eds.), Proc. 2nd INTEGRAL Workshop, "The Transparent Universe"

Teegarden B.J., Sturner S.J., 1999, in: Bazzano A. (ed.), Proc. 3rd INTEGRAL Workshop "The Extreme Universe"

Winkler C., 1997, in: Winkler C., Courvoisier T.J.-L. and Durouchoux P. (eds.), Proc. 2nd INTEGRAL Workshop, "The Transparent Universe" 